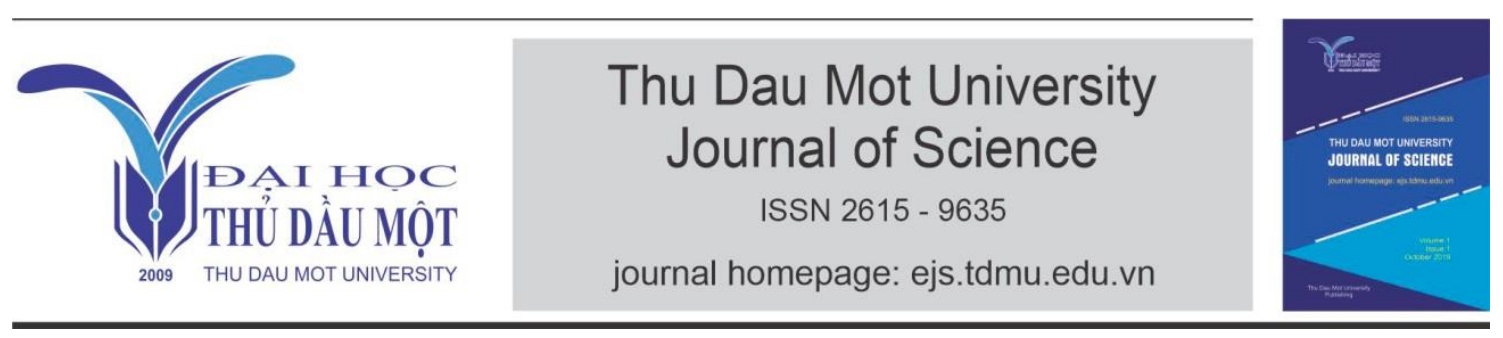

\title{
The nice $\boldsymbol{m}$-system of parameters for Artinian modules
}

by Nguyen Thi Khanh Hoa (Thu Dau Mot University)

Article Info: $\quad$ Received 02 Jan. 2020, Accepted 29 Feb. 2020, Available online 15 June. 2020 Corresponding author: hoanguyenthikhanh@gmail.com

https://doi.org/10.37550/tdmu.EJS/2020.02.042

\section{ABSTRACT}

This paper restates the definition of the nice $m$-system of parameters for Artinian modules. It also shows its effects on the differences between lengths and multiplicities of certain systems of parameters for Artinian modules:

$$
I(\underline{x}(\underline{n}) ; A)=\ell_{R}\left(O:_{A}\left(x_{1}^{n_{1}}, x_{2}^{n_{2}}, \ldots, x_{d}^{n_{d}}\right) R\right)-e\left(x_{1}^{n_{1}}, x_{2}^{n_{2}}, \ldots, x_{d}^{n_{d}} ; A\right)
$$

In particular, if $\underline{x}$ is a nice m-system of parameters then the function $I(\underline{x}(\underline{n}) ; A)$ is a polynomial having very nice form. Moreover, we will prove some properties of the nice m-system of parameters for Artinian modules. Especially, its effect on the annihilation of local homology modules of Artinian module A.

Keywords: annihilation, Artinian module, function of certain systems of parameters, local homology, nice m-system of parameters

\section{Introduction}

Throughout this paper, $(R, m)$ is a commutative Noetherian local ring with the maximal ideal $m$, and $A$ is an Artinian $R$-module with $\mathrm{N}-\operatorname{dim} A=d$.

+ The $R$-module $\underline{\underline{\lim }} \operatorname{Tor}_{i}^{R}\left(R / m^{t} ; A\right)$ is called ith-local homology module of $A$ with respect to $m$ and denoted by $H_{i}^{m}(A)$. 
+ Let $\underline{n}=\left(n_{l}, n_{2}, \ldots, n_{d}\right)$ be a d-tuple of positive integers. For each system of parameters (s.o.p) $\underline{x}=\left(x_{1}, x_{2}, \ldots, x_{d}\right)$ of $A$, we consider

$$
I(\underline{x}(\underline{n}) ; A)=\ell_{R}\left(O:_{A}\left(x_{1}^{n_{1}}, x_{2}^{n_{2}}, \ldots, x_{d}^{n_{d}}\right) R\right)-e\left(x_{1}^{n_{1}}, x_{2}^{n_{2}}, \ldots, x_{d}^{n_{d}} ; A\right)
$$

as a function d-variables on $n_{1}, n_{2}, \ldots, n_{d}$.

Let $I(A)=\sup _{x} I(\underline{x} ; A)$ where $\underline{x}$ runs over all s.o.p of A.

The value of function $I(\underline{x} ; A)$ and the annihilation of local homology modules of $A$ help us classify many different types of modules. Moreover, they also give us lots of information about different types of modules (see [3]). Such as:

$+I(A)=0: A$ is a co-Cohen-Macaulay module.

$+I(A)<\infty: A$ is a Generalized co-Cohen-Macaulay module.

$+I(\underline{x} ; A)$ is a constant for all s.o.p of $A: A$ is a co-Buchsbaum module.

+ If $A$ is a Generalized co-Cohen-Macaulay module, there exists an $m$-primary ideal $q$ such that $q H_{i}^{m}(A)=0$ for all $i=1, \ldots, d-1$.

+ If $A$ is a co-Buchsbaum module, $m H_{i}^{m}(A)=0$ for all $i=1, \ldots, d-1$.

However, $I(\underline{x}(\underline{n}) ; A)$ may be not a polynomial on $n_{l}, n_{2}, \ldots, n_{d}$ even when $n_{l}, n_{2}, \ldots, n_{d}$ large enough (see [1]), but [2] has shown that if $\underline{x}$ is a nice $m$-systems of parameters, $I(\underline{x}(\underline{n}) ; A)$ is a polynomial with simple form. In addition, a nice s.o.p of $A$ also annihilates local homology modules of $A$. Thus, in this paper we will restate the definition of the nice $m$-s.o.p for Artinian modules, the effect of the nice $m$-s.o.p on the calculation formula of function $I(\underline{x}(\underline{n}) ; A)$ and continue studying some its properties. Especially its effect on the annihilation of local homology modules of $A$.

\section{Preliminaries}

Lemma 2.1([1]). Assume $\left(A n n_{R} A\right) \hat{R}=A n n_{\hat{R}} A$ and $\underline{x}=\left(x_{1}, x_{2}, \ldots, x_{d}\right)$ is a s.o.p of Artinian R-module A. Then, there exits $j \in\{1,2, \ldots, d\}$ such that $x_{j}$ is a pseudo-Acoregular element. 
Nguyen Thi Khanh Hoa- Volume 2 - Issue 2-2020, p.158-165.

Lemma 2.2 ([3]). Let $x \in R$ be a pseudo-A-coregular element. Then $\ell_{R}(A / x A)<\infty$.

Lemma 2.3 ([4]). Let $M$ be an R-module, I be an ideal of $R$. Then for all $i \geq 0$,

$$
\bigcap_{s>0} I^{s} H_{i}^{I}(M)=0
$$

Lemma 2.4 ([1]). Let s a positive integer such that $m^{t} A=m^{s} A, \forall t \geq s$. Then

$$
H_{0}^{m}(A)=A / m^{s} A .
$$

Lemma 2.5. ([3]). For every s.o.p $\underline{x}$ of A, we have

$$
\ell_{R}\left(0:_{A} \underline{x} R\right)-e(\underline{x} ; A) \leq \sum_{i=0}^{d-1}\left(\begin{array}{c}
d-1 \\
i
\end{array}\right) \ell_{R}\left(H_{i}^{m}(A)\right) .
$$

Moreover, if $\ell_{R}\left(H_{i}^{m}(A)\right)<\infty$ for all $i<d$, then there exists an m-primary ideal $q$ such that the equality holds for every s.o.p $\underline{x}$ contained in $q$.

Definition 2.6 ([2]).

* The sequence $x_{1}, \ldots, x_{t} \in m$ is called an $m$-sequence for $A$ if:

(i) $x_{k} \notin \sum_{s \neq k} x_{s} R$ for all $k=1, \ldots, t$,

(ii) $x_{k}\left(0:_{A}\left(x_{1}, \ldots, x_{i-1}\right) R\right)=x_{k} x_{i}\left(0:_{A}\left(x_{1}, \ldots, x_{i-1}\right) R\right)$ for all $1 \leq i \leq k \leq t\left(x_{0}=0\right)$.

* The sequence $x_{1}, \ldots, x_{t} \in m$ is called a strong $m$-sequence for $A$ if $x_{1}^{n_{1}}, \ldots, x_{t}^{n_{t}}$ is $m$ sequence for all $\left(n_{1}, \ldots, n_{t}\right) \in \square^{t}$.

* A strong $m$-sequence $x_{1}, \ldots, x_{t} \in m$ is called a nice $m$-sequence for $A$ if:

(i) $t=1$; or

(ii) $t>1$ and $x_{1}, \ldots, x_{i-1}$ is a strong m-sequence of $0:_{A}\left(x_{i}^{n_{i}}, \ldots, x_{t}^{n_{t}}\right) R$ for all $2 \leq i \leq t$ and for all $n_{i}, \ldots, n_{t} \in \square$.

* A s.o.p for $A$ is called a nice m-s.o.p if it is a nice $m$-sequence.

Lemma 2.7 ([2]). Let $x_{1}, \ldots, x_{t}$ be an $m$-sequence for $A$. Then:

(i) $x_{i}\left(0:_{A}\left(x_{1}, \ldots, x_{i-1}\right) R\right)=x_{i}^{n}\left(0:_{A}\left(x_{1}, \ldots, x_{i-1}\right) R\right)$ for all $1 \leq i \leq t$ and $n \in \square$;

(ii) for every $(i, k)$ with $1 \leq i \leq k \leq t$ we have 


$$
x_{k}\left(O:_{A}\left(x_{1}, \ldots, x_{i-1}\right) R\right) \subseteq x_{i}\left(O:_{A}\left(x_{1}, \ldots, x_{i-1}\right) R\right)
$$

(iii) $x_{2}, \ldots, x_{t}$ is an m-sequence for $0:_{A} x_{1}$.

The following theorem shows that $I(\underline{x}(\underline{n}) ; A)$ will be a polynomial when $\underline{x}=\left(x_{1}, x_{2}, \ldots, x_{d}\right)$ is a nice $m$-s.o.p for $A$. Furthermore, in this case it has a nice form.

Theorem 2.8 ([2]). Let $\underline{x}=\left(x_{1}, x_{2}, \ldots, x_{d}\right)$ be a s.o.p for A. Then the following three conditions are equivalent:

(i) $\underline{x}$ is a nice m-s.o.p for A;

(ii) there exist non-negative intergers $\alpha_{0}(\underline{x}, A), \ldots, \alpha_{d-1}(\underline{x}, A)$ such that

$$
I(\underline{x}(\underline{n}) ; A)=\alpha_{0}(\underline{x}, A)+\sum_{i=1}^{d-1} n_{1} \ldots n_{i} \cdot \alpha_{i}(\underline{x}, A)
$$

for all $n_{1}, \ldots, n_{d} \geq 1$

(iii)

$$
I(\underline{x}(\underline{n}) ; A)=\ell_{R}\left(\frac{0:_{A}\left(x_{2}, \ldots, x_{d}\right) R}{x_{1}\left(0:_{A}\left(x_{2}, \ldots, x_{d}\right) R\right)}\right)+\sum_{i=1}^{d-1} n_{1} \ldots n_{i} \cdot e\left(x_{1}, \ldots, x_{i} ; \frac{0:_{A}\left(x_{i+2}, \ldots, x_{d}\right) R}{x_{i+1}\left(0:_{A}\left(x_{i+2}, \ldots, x_{d}\right) R\right)}\right)
$$

for all $n_{1}, \ldots, n_{d} \geq 1$.

\section{Main results}

In this section, we give some corollaries of Theorem 2.8 .

Corollary 3.1. Let $x_{1}, x_{2}, \ldots, x_{d}$ be a nice m-s.o.p for $A$ with $N$-dimA $=d \geq 2$. Then

i) For all $n_{1}, \ldots, n_{d} \in \square$ we have

$$
I\left(x_{1}^{n_{1}}, x_{2}^{n_{2}}, \ldots, x_{d}^{n_{d}} ; A\right)=I\left(x_{1}^{n_{1}}, x_{2}^{n_{2}}, \ldots, x_{d} ; A\right) .
$$

ii) For all $n_{2}, \ldots, n_{d} \in \square$ we have

$$
I\left(x_{2}^{n_{2}}, \ldots, x_{d}^{n_{d}} ; 0:_{A} x_{1}\right)=I\left(x_{1}, x_{2}^{n_{2}}, \ldots, x_{d}^{n_{d}} ; A\right) .
$$

Proof.

i) From (iii) of Theorem 2.8, we find that $I\left(x_{1}^{n_{1}}, x_{2}^{n_{2}}, \ldots, x_{d}^{n_{d}} ; A\right)$ doesn't depend on $n_{d}$. So we have 
Nguyen Thi Khanh Hoa- Volume 2 - Issue 2-2020, p.158-165.

$$
I\left(x_{1}^{n_{1}}, x_{2}^{n_{2}}, \ldots, x_{d}^{n_{d}} ; A\right)=I\left(x_{1}^{n_{1}}, x_{2}^{n_{2}}, \ldots, x_{d} ; A\right) .
$$

ii) For any $\left(n_{2}, \ldots, n_{d}\right) \in \square^{d-1}$, we have

$$
I\left(x_{2}^{n_{2}}, \ldots, x_{d}^{n_{d}} ; 0:_{A} x_{1}\right)=I\left(x_{1}, x_{2}^{n_{2}}, \ldots, x_{d}^{n_{d}} ; A\right)-e\left(x_{2}^{n_{2}}, \ldots, x_{d}^{n_{d}} ; A / x_{1} A\right) .
$$

Because $x_{1}, x_{2}, \ldots, x_{d}$ is an $m$-sequence, by Lemma 2.7 we have $x_{2}^{n_{2}} A \subseteq x_{2} A \subseteq x_{1} A$. Hence, $x_{2}^{n_{2}}\left(A / x_{1} A\right)=0$. So that $e\left(x_{2}^{n_{2}}, \ldots, x_{d}^{n_{d}} ; A / x_{1} A\right)=0$.

This deduce $I\left(x_{2}^{n_{2}}, \ldots, x_{d}^{n_{d}} ; 0:_{A} x_{1}\right)=I\left(x_{1}, x_{2}^{n_{2}}, \ldots, x_{d}^{n_{d}} ; A\right)$.

Next, we give some example for nice $m$-s.o.p.

Remark 3.2.

i) Let $A$ be an co-Cohen-Macaulay R-module. Then every s.o.p of $A$ is a nice $m$ s.o.p.

ii) Let $A$ be an co-Buchsbaum R-module. Then every s.o.p of $A$ is a nice m-s.o.p.

iii) Let $A$ be an generalized co-Cohen-Macaulay R-module. Then there exists an $m$-primary ideal $q$ such that every s.o.p contain in $q$ is a nice $m$-s.o.p.

Proof.

i) As $A$ is an co-Cohen-Macaulay module, $I(A)=\sup _{\underline{x}} I(\underline{x} ; A)=0$ with $\underline{x}$ run over all s.o.p of $A$. From Theorem 2.8, we get $\underline{x}$ is a nice $m$-s.o.p.

ii) As $A$ is an co-Buchsbaum module, $I(\underline{x} ; A)$ is a constant (not depending on s.o.p $\underline{x}$ of $A$ ). From Theorem 2.8, we get $\underline{x}$ is a nice $m$-s.o.p.

iii) As $A$ is an generalized co-Cohen-Macaulay $R$-module, $\ell_{R}\left(H_{i}^{m}(A)\right)<\infty$ for all $i<d$. Thus, from Lemma 2.5 and Theorem 2.8, there exists an $m$-primary ideal $q$ such that every s.o.p contain in $q$ is a nice $m$-s.o.p.

Finally, we continue studying the effect of a nice $m$-s.o.p on the annihilation of local homology modules of $A$.

Proposition 3.3. Assume $\left(A n n_{R} A\right) \hat{R}=A n n_{\hat{R}} A$ and $\underline{x}=\left(x_{1}, x_{2}, \ldots, x_{d}\right)$ is a s.o.p and a strong $m$-sequence of Artinian R-module A. Then

$$
x_{j} H_{i}^{m}(A)=0 \text { for all } 0 \leq i<j \leq d .
$$


Proof. We proceed by introduction on $d=\mathrm{N}-\operatorname{dim} A$.

For $d=1$ and let $x_{1}$ be a s.o.p of $A$. Because of $A$ is an Artinian $R$-module, the system $\left\{m^{t} A\right\}$ is stationary, i.e there exists a positive interger $s$ such that $m^{t} A=m^{s} A$, for all $t \geq s$.

It follows from Lemma 2.4 that $x_{1} H_{0}^{m}(A)=x_{1} A / m^{s} A$.

Since $x_{1}$ is $m$-sequence for $A$ and $x_{1} A=x_{1}^{s} A$, we have $x_{1} A \subset m^{s} A$. This implies $x_{1} H_{0}^{m}(A)=0$.

Assume that $d>1$ and our assertion is true for all Artinian $R$-module of $\mathrm{N}$-dim smaller than $d$.

First, we shall prove $x_{j} H_{0}^{m}(A)=0$ for all $1 \leq j \leq d$. Similar proof in case $d=1$, from Lemma 2.7, we get $x_{j} A \subset x_{1} A=x_{1}^{s} A \subset m^{s} A$.

Next, we shall prove $x_{j} H_{i}^{m}(A)=0$ for all $1 \leq i<j \leq d$.

According to Lemma 2.1 and Lemma 2.2, there exists $k \in\{1, \ldots, d\}$ such that $x_{k}$ is a pseudo- $A$-coregular element and $\ell_{R}\left(A / x_{k} A\right)<\infty$. Since $x_{1}, x_{2}, \ldots, x_{d}$ is a s.o.p and a strong $m$-sequence of $A$, we have $x_{k} A \subset x_{1} A$. Thus $\ell_{R}\left(A / x_{1} A\right)<\ell_{R}\left(A / x_{k} A\right)<\infty$. This deduces that $\mathrm{N}-\operatorname{dim}\left(A / x_{1} A\right) \leq 0$. So $H_{i}^{m}\left(A / x_{1} A\right)=0$ for all $i>0$.

The exact sequence $0 \rightarrow x_{1} A \rightarrow A \rightarrow A / x_{1} A \rightarrow 0$ generates the long exact sequence

$$
\cdots \rightarrow H_{i+1}^{m}\left(A / x_{1} A\right) \rightarrow H_{i}^{m}\left(x_{1} A\right) \rightarrow H_{i}^{m}(A) \rightarrow H_{i}^{m}\left(A / x_{1} A\right) \rightarrow \cdots
$$

Since $H_{i+1}^{m}\left(A / x_{1} A\right)=H_{i}^{m}\left(A / x_{1} A\right)=0$ we have $H_{i}^{m}\left(x_{1} A\right) \cong H_{i}^{m}(A)$ for all $i>0$. Moreover, because $x_{1}, x_{2}, \ldots, x_{d}$ is an $m$-sequence of $A$, we get $x_{1} A=x_{1}^{n} A$ for all $n>0$. This deduces

$$
H_{i}^{m}(A) \cong H_{i}^{m}\left(x_{1} A\right) \cong H_{i}^{m}\left(x_{1}^{n} A\right) \text { for all } i, n>0
$$

Combining this result and the exact sequence $0 \rightarrow 0:_{A} x_{1}^{n} \rightarrow A \rightarrow x_{1}^{n} A \rightarrow 0$ we have the long exact sequence:

$$
\cdots \rightarrow H_{i}^{m}(A) \stackrel{x_{1}^{n}}{\longrightarrow} H_{i}^{m}(A) \stackrel{\Delta_{i}}{\longrightarrow} H_{i-1}^{m}\left(0:_{A} x_{1}^{n}\right) \rightarrow H_{i-1}^{m}(A) \stackrel{x_{1}^{n}}{\longrightarrow} H_{i-1}^{m}(A) \rightarrow \cdots
$$


Nguyen Thi Khanh Hoa- Volume 2 - Issue 2-2020, p.158-165.

Since $\operatorname{Ker}_{i}=\operatorname{Im} x_{1}^{n}=x_{1}^{n} H_{i}^{m}(A), \forall n>0$ we have $\operatorname{Im} \Delta_{i} \cong \frac{H_{i}^{m}(A)}{\operatorname{Ker} \Delta_{i}}=\frac{H_{i}^{m}(A)}{x_{1}^{n} H_{i}^{m}(A)}$.

As $x_{1}, x_{2}, \ldots, x_{d}$ is a strong $m$-sequence of $A$ then $x_{2}, \ldots, x_{d}$ is a strong $m$-sequence of $0:_{A} x_{1}^{n}$. Applying the inductive hypothesis for $0:_{A} x_{1}^{n}$ to have $x_{j} H_{i-1}^{m}\left(0:_{A} x_{1}^{n}\right)=0$ for all $1 \leq i<j \leq d$. Therefore $x_{j} \operatorname{Im} \Delta_{i}=0$. Combining this result and Lemma 2.3 we get

$$
x_{j} H_{i}^{m}(A) \subseteq x_{1}^{n} H_{i}^{m}(A) \subseteq m^{n} H_{i}^{m}(A), \forall n>0 \Rightarrow x_{j} H_{i}^{m}(A) \subseteq \bigcap_{n>0} m^{n} H_{i}^{m}(A)=0 .
$$

Our proof is complete.

Corollary 3.4. Assume $\left(A n n_{R} A\right) \hat{R}=A n n_{\hat{R}} A$.

i) Let $x_{1}, x_{2}, \ldots, x_{d}$ be a s.o.p and strong $m$-sequence of $A$. Then

$$
x_{j} H_{i}^{m}\left(0:_{A}\left(x_{1}^{n_{1}}, \ldots, x_{k}^{n_{k}}\right)\right)=0 \text { for all } 0 \leq i, k<j \leq d .
$$

ii) Let $x_{1}, x_{2}, \ldots, x_{d}$ be a nice m-s.o.p of A. Then

$$
x_{j} H_{i}^{m}\left(0:_{A}\left(x_{k}^{n_{k}}, \ldots, x_{d}^{n_{d}}\right)\right)=0 \text { for all } 0 \leq i<j<k \leq d .
$$

Proof.

i) Because $x_{1}, x_{2}, \ldots, x_{d}$ is a s.o.p and a strong $m$-sequence of $A$, $x_{1}^{n_{1}}, \ldots, x_{k}^{n_{k}}, x_{k+1}^{n_{k+1}}, \ldots, x_{d}^{n_{d}}$ is also a s.o.p and an $m$-sequence of $A$ for all $n_{1}, n_{2}, \ldots, n_{d} \in \square$. Therefore $x_{k+1}, \ldots, x_{d}$ is a s.o.p and a strong $m$-sequence of $0:_{A}\left(x_{1}^{n_{1}}, \ldots, x_{k}^{n_{k}}\right)$.

By Proposition 3.3, we have $x_{j} H_{i}^{m}\left(0:_{A}\left(x_{1}^{n_{1}}, \ldots, x_{k}^{n_{k}}\right)\right)=0$ for all $0 \leq i, k<j \leq d$.

ii) Because $x_{1}, x_{2}, \ldots, x_{d}$ is a nice s.o.p, $x_{1}, \ldots, x_{k-1}$ is also a s.o.p and a strong $m$ sequence of $0:_{A}\left(x_{k}^{n_{k}}, \ldots, x_{d}^{n_{d}}\right)$ for all $n_{k}, \ldots, n_{d} \in \square$.

By Proposition 3.3, we have $x_{j} H_{i}^{m}\left(0:_{A}\left(x_{k}^{n_{k}}, \ldots, x_{d}^{n_{d}}\right)\right)=0$. 


\section{References}

N.D. Minh (2006). Least degree of polynomials certain systems of parameters for Artinian modules. Southeast Asian Bulletin of math, 30, 85-97.

N.D. Minh, N.T.K. Hoa, T.T.Nam (2014). On polynomial property of a function certain systems of parameters for Artinian modules. Kyushu Journal of Math, 68(2), 239-248.

N.T. Cuong, N.T.Dung and L.N. Nhan (2007). Generalized co-Cohen-Macaulay and coBuchsbaum modules, Algebra Colloquium, Vol. 14, 265.

N.T. Cuong and T.T. Nam (2001). The I-adic completion and local homology for Artinian modules. Math.Proc. Cambridge Philos. Soc. 131(1), 61-72. 